\title{
Perfil cognitivo dos alunos com dificuldades de aprendizagem na leitura e matemática
}

\author{
Luciana Vellinho Corso' \\ Beatriz Vargas Dorneles \\ Universidade Federal do Rio Grande do Sul, Porto Alegre - RS - Brasil
}

\begin{abstract}
Resumo: A coocorrência entre as dificuldades na leitura e na matemática é frequente, indicando que as habilidades cognitivas comuns subjacentes a tais dificuldades possam estar deficitárias. A pesquisa aqui descrita analisa as habilidades cognitivas que estão na base das aprendizagens da leitura e da matemática, contribuindo para a prática pedagógica nessas áreas. Trata-se de um estudo transversal de caráter quantitativo em que foram identificadas funções cognitivas de 79 alunos do $3^{\circ}$ ao $6^{\circ}$ ano do ensino fundamental, divididos em quatro grupos: com dificuldades na leitura, na matemática, nas duas áreas e sem dificuldades. Obtivemos o perfil cognitivo dos grupos com tarefas de processamento fonológico, senso numérico, memória de trabalho e estratégias de contagem e recuperação da memória. Cada grupo apresentou um perfil cognitivo único, indicando necessidades educacionais diversas. Os resultados alertam para a importância da estimulação do processamento fonológico e do senso numérico, habilidades ainda pouco conhecidas pela escola brasileira.
\end{abstract}

Palavras-chave: dificuldades de aprendizagem; leitura; matemática; habilidades cognitivas; perfil cognitivo.

\section{COGNITIVE PROFILE OF STUDENTS WITH LEARNING DIFFICULTIES IN READING AND MATHEMATICS}

\begin{abstract}
The co-occurrence of difficulties in reading and mathematics is frequent, indicating that common cognitive skills underlying these difficulties may be impaired. The study aims to analyse the cognitive abilities which are in the basis of acquisition of both reading and mathematics skills contributing to the educational practice in these areas. This is a cross-sectional, quantitative study that identified cognitive functions of 79 students from 3rd to 6th grade of elementary school. The students were divided into four groups: difficulties in reading, in mathematics, in both areas and the control group. We assessed the cognitive profile of the students through tasks involving: phonological processing, number sense, working memory, counting strategies and arithmetic fact retrieval. Each group showed a unique cognitive profile indicating different educational needs. The results urge attention to the importance of the stimulation of phonological processing and number sense, abilities which are still unknown by Brazilian schools.
\end{abstract}

Keywords: learning difficulties; reading; mathematics; cognitive abilities; cognitive profile.

1 Endereço para correspondência: Luciana Vellinho Corso, Faculdade de Educação da Universidade Federal do Rio Grande do Sul. Departamento de Estudos Especializados, Av. Paulo Gama, 110, prédio 12201, $9^{\circ}$ andar, sala 918, Farroupilha - Rio Grande do Sul - RS - Brasil. CEP: 90046-060. E-mail: I.corso@terra.com.br. 


\begin{abstract}
Resumen: La co-ocurrencia de dificultades en lectura y matemática son frecuentes, lo que indica que habilidades cognitivas comunes que subyacen a estas dificultades pueden ser deficientes. La investigación descrita aquí examina las habilidades cognitivas que subyacen en el aprendizaje de lectura y matemática, lo que contribuye a la práctica pedagógica en estas áreas. Se trata de un estudio transversal, de carácter cuantitativo, en el que se identificaron las funciones cognitivas de los 79 estudiantes de $3^{\circ}$ al $6^{\circ}$ año de la escuela primaria, divididos en cuatro grupos: con dificultades en lectura, en matemática, en las dos áreas y sin dificultades. Se ha observado el perfil cognitivo de los grupos con tareas de procesamiento fonológico, sentido numérico, memoria de trabajo y estrategias para contar y recuperación de la memoria. Cada grupo presentó un perfil cognitivo único que indica diversas necesidades educativas. Los resultados ponen de relieve la importancia de la estimulación del procesamiento fonológico y el sentido numérico, habilidades estas aún poco conocidas por la escuela brasileña.
\end{abstract}

Palabras clave: problemas de aprendizaje; lectura; matemática; habilidades cognitivas; perfil cognitivo.

A coexistência de dificuldades na leitura e na matemática é frequente. Os estudos que tratam desse tema indicam possíveis relações existentes entre essas áreas. A literatura sugere que as dificuldades na leitura e na matemática e a coexistência dessas dificuldades podem estar associadas a deficiências em uma ou mais habilidades cognitivas, como processamento fonológico (consciência fonológica, memória fonológica e velocidade de processamento), senso numérico, memória de trabalho (executivo central) e estratégias e procedimentos de contagem e de recuperação de fatos da memória (Geary, Hamson, \& Hoard, 2000). Pesquisas recentes têm investigado as bases orgânicas de tais habilidades (Ashkenazi, Black, Black, Hoeft, \& Menon, 2013) e apontam que, para buscar formas efetivas de intervir, é necessária uma maior compreensão dos fatores cognitivos e neurobiológicos que contribuem para os altos níveis de comorbidade nas áreas de leitura e matemática.

O estudo que apresentamos investigou tais habilidades cognitivas em crianças brasileiras. O objetivo central da pesquisa foi compreender as habilidades cognitivas que subjazem à aprendizagem da leitura e da matemática, trazendo, consequentemente, avanços para a prática pedagógica nessas áreas.

\title{
Perfil cognitivo dos alunos com dificuldades na leitura
}

A seguir, descrevemos as habilidades de processamento fonológico mais diretamente ligadas às dificuldades na leitura (DL).

- Consciência fonológica: está inserida nos conhecimentos metalinguísticos que permitem fazer da língua um objeto de pensamento, de modo a possibilitar a reflexão sobre os sons da fala, o julgamento e a manipulação da estrutura sonora das palavras. Tal consciência desenvolve-se gradualmente a partir de habilidades existentes antes do início da aquisição da escrita. Essas habilidades que são aprimoradas com o tempo contribuem para o surgimento de novas habilidades metafonológicas 
(Shaywitz, 2006). Hoje, existe um relativo consenso de que as dificuldades em leitura podem ser causadas por déficits nas habilidades de processamento fonológico, principalmente a consciência fonológica, já que tais déficits interferem diretamente na aquisição e no domínio das correspondências letra-som necessárias para uma leitura fluente (Hecht, Torgesen, Wagner, \& Rashotte, 2001; Willcutt et al., 2013).

- Velocidade de processamento: refere-se à eficiência com que tarefas cognitivas simples são executadas. Na literatura, encontramos tarefas diversas utilizadas como medidas de velocidade de processamento, tais como tarefas de velocidade de acesso que avaliam a velocidade com que as informações fonológicas da memória de longo prazo são acessadas (Hecht et al., 2001). Há evidências de que quanto mais rapidamente a criança consegue nomear séries de letras ou números (capacidade de buscar as informações verbais na memória de longo prazo), um melhor desempenho é apresentado na sua leitura (Cardoso-Martins \& Pennington, 2001; Willcutt et al., 2013). Assim, a velocidade de processamento tem sido apontada como uma boa preditora de sucesso em leitura.

- Memória de trabalho (componente executivo central): trata-se de um sistema cognitivo responsável pelo armazenamento e processamento simultâneo da informação. No modelo de Baddeley e Hitch (1974), três componentes formam esse sistema: executivo central, componente fonológico e visuoespacial. Os estudos sobre a memória de trabalho, além de destacarem a importância do componente fonológico, têm apontado o executivo central como aquele que desempenha um importante papel no aprendizado da leitura (Swanson, 1999). Uma relação entre a habilidade na leitura e o funcionamento do executivo central parece compreensível, particularmente nos estágios em que a habilidade da leitura não está completamente automatizada. Nesse período, para o novo leitor, o processo de decodificação da palavra é ainda uma tarefa dispendiosa porque os segmentos de palavras ou sentenças necessitam ser memorizados enquanto os segmentos restantes são decodificados.

- Memória fonológica: compreende aquele aspecto da memória de trabalho que envolve armazenar temporariamente as representações de som. Quando uma criança lê uma frase, é preciso guardar na mente várias unidades de informação para juntá-las e entender o que acabou de ler. Estudos apontam que alunos com DL apresentam baixa capacidade de memória fonológica (Swanson, 1999).

\section{Perfil cognitivo dos alunos com dificuldades na matemática}

Apresentamos, a seguir, uma visão geral sobre o desempenho de alunos com dificuldades na matemática (DM), em algumas habilidades cognitivas.

- Procedimentos aritméticos: durante a aprendizagem inicial da adição, as crianças tipicamente contam ambas as parcelas utilizando o procedimento de "contar todos". Depois, passam a utilizar o "contar a partir de" (contar a partir de uma das parcelas dadas). Com a prática e a internalização desses procedimentos, evidencia-se o surgimento de outras formas de resolução das situações-problema: a recuperação 
de fatos da memória $(5+3=8)$ e a decomposição $(6+7=6+6+1)$, estratégias mais sofisticadas e econômicas. Já foram descritas diferenças no desempenho de alunos com e sem DM, no que diz respeito à resolução de problemas aritméticos simples (por exemplo, $4+3=7$ ). Observou-se que aqueles alunos com dificuldades cometem mais erros de contagem e utilizam os procedimentos imaturos de "contar todos" mais frequentemente do que os sem dificuldades (Orrantia, Martínez, Morán, \& Fernández, 2002).

- Senso numérico: de modo geral, o senso numérico refere-se à facilidade e flexibilidade com números e à compreensão do significado dos números e das ideias relacionadas a eles. Os alunos com DM evidenciam um senso numérico pouco desenvolvido (Gersten, Jordan, \& Flojo, 2005; Dyson, Jordan, \& Glutting, 2011). Este, por sua vez, pode se dever a uma representação e/ou a um processamento imaturo dos números que ocasiona defasagens na compreensão e flexibilidade no senso numérico e acarreta problemas para o desenvolvimento de habilidades como contagem, realização de operações, estimativas e cálculo mental.

- Memória de trabalho: trata-se de uma habilidade cognitiva frequentemente citada na literatura como prejudicada nos alunos com DM. Há indicadores de uma forte ligação entre a capacidade de memória de trabalho e habilidades cognitivas superiores, tais como aritmética (Hecht et al., 2001) e leitura e compreensão (Swanson, 1999). Também há um conjunto de evidências que indicam que a memória de trabalho está relacionada a diferentes processos (Gersten et al., 2005): retenção de números durante o processo aritmético (componente fonológico), representação espacial de problemas multidígitos (componente visuoespacial) e direcionamento e monitoramento de procedimentos em problemas aritméticos complexos (componente executivo central).

- Velocidade de processamento: já é bem estabelecida a correlação entre a velocidade de processamento e as habilidades de cálculo (Hecht et al., 2001). Crianças com DM processam a informação mais lentamente do que seus pares sem dificuldades (Murphy, Mazzocco, Hanich, \& Early, 2007). Estudos como os de Geary et al. (2000) evidenciam que os alunos com DM são mais lentos para realizar as estratégias de contagem do que os colegas com desenvolvimento típico. A velocidade de processamento dita o quão rapidamente os números podem ser contados. Portanto, ela pode facilitar a velocidade de contagem de forma que, à medida que o aluno ganha velocidade na contagem de conjuntos para descobrir as somas e diferenças, os problemas são sucessivamente associados às suas respostas na memória de trabalho antes de se perderem, de modo que aquela associação pode ser estabelecida na memória de longo prazo. A velocidade de processamento está subjacente à fluência em fatos aritméticos, e, por isso, autores como Hopkins e Lawson (2006) identificam tal habilidade como um determinante crítico do desenvolvimento da recuperação de fatos da memória.

- Memória semântica de longo prazo: a inabilidade para desenvolver, fortalecer e acessar associações na memória de longo prazo que permitam a recuperação rápida 
e acurada de respostas a problemas básicos de adição é uma característica das DM (Hopkins \& Lawson, 2006). De acordo com Orrantia et al. (2002), a dificuldade na recuperação de fatos se relaciona com os seguintes fatores: enfraquecimento da informação na memória de trabalho, velocidade lenta na execução de estratégias de contagem e alta frequência de erros de contagem. Com uma velocidade de contagem lenta, existe maior probabilidade de esquecimento da informação na memória de trabalho, o que leva ao não desenvolvimento de representações na memória.

Em síntese, podemos dizer que os processos cognitivos descritos são fundamentais para o bom desempenho nas áreas de leitura e de matemática e podem estar prejudicados nos alunos que enfrentam problemas nessas áreas. Pesquisas apontam que, de modo geral, os alunos com a coexistência de DL + DM apresentam dificuldades mais severas e de funcionamento global do que os alunos com dificuldades em apenas uma dessas áreas. O estudo de Willcutt et al. (2013), ao comparar o desempenho de diferentes grupos de crianças (com DL, com DM, com DL + DM e sem dificuldades), apontou problemas significativos na memória de trabalho, na velocidade de processamento e na compreensão verbal de crianças com a coexistência de DL + DM, enquanto, no grupo de crianças com DL apenas, os problemas mostraram-se restritos às habilidades de consciência fonológica e velocidade de nomeação. Por isso, os pesquisadores da área chamam a atenção para a importância de se fazer pesquisa considerando a distinção existente entre alunos com dificuldades específicas na matemática e alunos com dificuldades coexistentes na matemática e na leitura (Andersson \& Lyxell, 2007).

\section{Método}

\section{Participantes}

Participaram desta pesquisa 79 alunos do $3^{\circ}$ ao $6^{\circ}$ ano do ensino fundamental, provenientes de cinco escolas estaduais de Porto Alegre (RS), com ou sem dificuldades nas áreas de leitura e/ou matemática. Foram utilizados três critérios para a composição do grupo experimental: indicação do professor, desempenho nos subtestes de leitura e matemática do teste de desempenho escolar (TDE) de Stein (1994) e desempenho nas subprovas de cubos e vocabulário da escala Wechsler de inteligência para crianças (Wechsler intelligence scale for children - Wisc). Tais provas foram administradas para que evitássemos trabalhar com alunos com baixo rendimento intelectual. Foi solicitada a autorização dos pais, que assinaram o Termo de Consentimento Livre e Esclarecido, para que os alunos participassem da pesquisa. O grupo experimental foi classificado em quatro subgrupos: 1. alunos com (DL), 2. alunos com (DM), 3. alunos com (DL + DM) e 4. alunos sem dificuldades (CONT). A Tabela 1 sumariza os dados referentes aos quatro grupos participantes da pesquisa. Para este estudo, definimos como alunos com DL e DM aqueles que apresentam desempenho acadêmico abaixo do que seria esperado para o seu nível de escolaridade naquelas áreas, considerando os resultados nas tarefas avaliativas utilizadas. 
Tabela I. Número de participantes, gênero, média de idade e desvio padrão dos diferentes grupos

\begin{tabular}{lccccccc} 
Grupo & N & Meninos & Meninas & $\begin{array}{c}\text { Idade } \\
\text { mínima }\end{array}$ & $\begin{array}{c}\text { Idade } \\
\text { máxima }\end{array}$ & $\begin{array}{c}\text { Média } \\
\text { de idade }\end{array}$ & $\begin{array}{c}\text { Desvio } \\
\text { padrão }\end{array}$ \\
\hline DL & 20 & 11 & 9 & 10 anos & $\begin{array}{l}14 \text { anos e } \\
3 \text { meses }\end{array}$ & $\begin{array}{c}11 \text { anos e } \\
10 \text { meses }\end{array}$ & 1,36 \\
\hline DM & 13 & 8 & 5 & $\begin{array}{l}10 \text { anos e } \\
11 \text { meses }\end{array}$ & $\begin{array}{l}17 \text { anos e } \\
7 \text { meses }\end{array}$ & $\begin{array}{l}13 \text { anos e } \\
7 \text { meses }\end{array}$ & 1,70 \\
\hline DLM & 25 & 12 & 13 & $\begin{array}{l}10 \text { anos e } \\
3 \text { meses }\end{array}$ & $\begin{array}{l}14 \text { anos e } \\
7 \text { meses }\end{array}$ & $\begin{array}{l}11 \text { anos e } \\
9 \text { meses }\end{array}$ & 1,43 \\
\hline CONT & 21 & 12 & 9 & 10 anos e & $\begin{array}{l}13 \text { anos e } \\
4 \text { meses }\end{array}$ & $\begin{array}{l}11 \text { anos e } \\
8 \text { meses }\end{array}$ & 1,10 \\
\hline
\end{tabular}

DL: alunos com dificuldades na leitura; DM: alunos com dificuldades na matemática; DLM: alunos com dificuldades na leitura e na matemática; CONT: alunos sem dificuldades de aprendizagem.

Fonte: Corso (2008).

\section{Instrumentos utilizados}

- Processamento fonológico: consciência fonológica: instrumento de avaliação sequencial - Confias (Moojen et al., 2003); memória fonológica para dígitos, frases e relatos (adaptada de Golbert, 1988); velocidade de acesso a informações da memória de longo prazo: nomeação de dígitos, letras e dígitos e letras (tarefa adaptada de Hecht et al., 2001).

- Senso numérico: teste de conhecimento numérico (Okamoto \& Case, 1996).

- Memória de trabalho (componente executivo central): memória de trabalho $1 \mathrm{com}$ informação não numérica (adaptada de Hecht et al., 2001) e memória de trabalho 2 com informação numérica (tarefa adaptada de Yuill, Oakhill, \& Parkin, 1989).

- Estratégias e procedimentos de contagem e de recuperação da memória: tarefas de Geary et al. (2000) para avaliar as estratégias utilizadas na realização de cálculos de adição simples (tarefa 1) e o uso da estratégia de recuperação da memória (tarefa 2).

\section{Procedimentos}

O subteste de matemática do TDE foi aplicado coletivamente em sala de aula, dentro do horário escolar, pelo pesquisador. O subteste de leitura do TDE e todas as demais tarefas foram aplicados individualmente pelo pesquisador. A testagem da Wisc foi feita por psicólogo.

\section{Análise dos dados}

Utilizamos a análise de variância, Anova (oneway) com post hoc de Tukey, no nível de significância de $p<0,05$, para evidenciarmos as diferenças existentes entre os quatro grupos participantes da pesquisa em relação às habilidades avaliadas. 


\section{Resultados}

As médias e os desvios padrão obtidos pelos quatro grupos nas tarefas de processamento fonológico estão presentes na Tabela 2.

\section{Tabela 2. Desempenho obtido pelos diferentes grupos nas tarefas de processamento fonológico}

\begin{tabular}{|c|c|c|c|c|c|c|c|c|c|}
\hline \multirow[b]{2}{*}{ Tarefa } & \multicolumn{2}{|c|}{$\begin{array}{c}\mathrm{DL} \\
(\mathrm{n}=20)\end{array}$} & \multicolumn{2}{|c|}{$\begin{array}{c}D M \\
(n=13)\end{array}$} & \multicolumn{2}{|c|}{$\begin{array}{c}\text { DLM } \\
(n=25)\end{array}$} & \multicolumn{2}{|c|}{$\begin{array}{c}\text { CONT } \\
(n=2 \mathrm{I})\end{array}$} & \multirow[b]{2}{*}{ P-Valor } \\
\hline & M & DP & M & DP & M & DP & M & DP & \\
\hline Consciência da sílaba & 24,70 & $2,43 a b$ & 25,00 & $2,97 a b$ & 24,00 & $2,64 a b$ & 25,62 & 3,23ab & 0,284 \\
\hline Consciência do fonema & 18,50 & $3,13 a$ & 19,69 & $4,07 a b$ & 17,92 & $3,45 a$ & 21,90 & $3,49 b$ & 0,002 \\
\hline Consciência total & 43,20 & $4,25 a$ & 44,69 & $6,56 \mathrm{ab}$ & 41,92 & $5,46 a$ & 48,00 & $5,65 b$ & 0,003 \\
\hline Memória de dígitos & 9,20 & $\mathrm{I}, 57 \mathrm{ab}$ & 10,00 & $\mathrm{I}, 58 \mathrm{ab}$ & 9,24 & $\mathrm{I}, 73 \mathrm{ab}$ & 9,90 & $\mathrm{I}, 8 \mathrm{I}$ & 0,326 \\
\hline Memória de frases & 9,08 & $0,84 a b$ & 9,29 & $0,37 a b$ & 9,13 & $0,60 a b$ & 9,29 & 0,36 & 0,591 \\
\hline Memória de relatos & 22,20 & $3,33 a b$ & 18,85 & $5,97 a$ & 21,68 & $5,39 a b$ & 23,43 & $3,20 b$ & 0,46 \\
\hline Velocidade números & 14,75 & $2,40 b$ & 12,95 & $\mathrm{I}, 6 \mathrm{I} a \mathrm{~b}$ & 14,37 & $2,63 b$ & 11,89 & $2,02 a$ & 0,000 \\
\hline Velocidade letras & 14,08 & $\mathrm{I}, 87 \mathrm{ab}$ & 12,54 & $2,09 a$ & 14,84 & $3,3 \mathrm{Ib}$ & 11,99 & $2,14 a$ & 0,001 \\
\hline Velocidade números e letras & 16,84 & $2,25 b c$ & 15,35 & $2,36 a b$ & 18,16 & $3,20 c$ & 13,94 & $2,20 \mathrm{a}$ & 0,000 \\
\hline
\end{tabular}

DL: alunos com dificuldades na leitura; DM: alunos com dificuldades na matemática; DLM: alunos com dificuldades na leitura e na matemática; CONT: alunos sem dificuldades de aprendizagem.

* Letras iguais indicam que as médias não diferem.

Fonte: Corso (2008).

Os resultados apresentados na Tabela 3 mostram o desempenho dos diferentes grupos em senso numérico.

Tabela 3. Desempenho obtido pelos diferentes grupos na tarefa de senso numérico

\begin{tabular}{|c|c|c|c|c|c|c|c|c|c|}
\hline & \multicolumn{2}{|c|}{$\begin{array}{c}D L \\
(n=20)\end{array}$} & \multicolumn{2}{|c|}{$\begin{array}{c}D M \\
(n=13)\end{array}$} & \multicolumn{2}{|c|}{$\begin{array}{c}\text { DLM } \\
(n=25)\end{array}$} & \multicolumn{2}{|c|}{$\begin{array}{c}\text { CONT } \\
(n=2 I)\end{array}$} & \multirow[b]{2}{*}{ P-valor } \\
\hline & $M$ & DP & $M$ & DP & $M$ & DP & $M$ & DP & \\
\hline $\begin{array}{l}\text { Senso } \\
\text { numérico }\end{array}$ & 38,80 & $4,44 a b$ & 38,00 & $6,37 a b$ & 35,92 & $5,84 a$ & 41,43 & $4,73 b$ & 0,009 \\
\hline
\end{tabular}

DL: alunos com dificuldades na leitura; DM: alunos com dificuldades na matemática; DLM: alunos com dificuldades na leitura e na matemática; CONT: alunos sem dificuldades de aprendizagem.

* Letras iguais indicam que as médias não diferem.

Fonte: Corso (2008). 
A Tabela 4 mostra os resultados evidenciados pelos grupos nas tarefas de memória de trabalho 1 (informação não numérica) e 2 (informação numérica).

Tabela 4. Desempenho obtido pelos diferentes grupos nas tarefas de memória de trabalho (componente executivo central)

\begin{tabular}{|c|c|c|c|c|c|c|c|c|c|}
\hline & \multicolumn{2}{|c|}{$\begin{array}{c}D L \\
(n=20)\end{array}$} & \multicolumn{2}{|c|}{$\begin{array}{c}D M \\
(n=13)\end{array}$} & \multicolumn{2}{|c|}{$\begin{array}{c}\text { DLM } \\
(n=25)\end{array}$} & \multicolumn{2}{|c|}{$\begin{array}{c}\text { CONT } \\
(n=21)\end{array}$} & \multirow[b]{2}{*}{ P-valor } \\
\hline & $M$ & DP & $M$ & DP & $M$ & DP & $M$ & DP & \\
\hline Tarefa I & 26,00 & $3,06 a b$ & 23,92 & $3,94 a b$ & 23,20 & $3,83 a$ & 26,19 & $2,60 b$ & 0,008 \\
\hline Tarefa 2 & 7,85 & I,89ab & 8,62 & I,89ab & 7,16 & $2,03 a$ & 9,29 & $2,8 \mathrm{Ib}$ & 0,014 \\
\hline
\end{tabular}

DL: alunos com dificuldades na leitura; DM: alunos com dificuldades na matemática; DLM: alunos com dificuldades na leitura e na matemática; CONT: alunos sem dificuldades de aprendizagem.

* Letras iguais indicam que as médias não diferem.

Fonte: Corso (2008).

Os resultados alcançados pelos grupos quanto ao uso das diferentes estratégias encontram-se na Tabela 5.

Tabela 5. Média e desvio padrão da frequência de uso espontâneo das estratégias de contar nos dedos, contagem interna, decomposição e recuperação da memória

\begin{tabular}{|c|c|c|c|c|c|c|c|c|c|}
\hline & \multicolumn{2}{|c|}{$\begin{array}{c}\mathrm{DL} \\
(\mathrm{n}=20)\end{array}$} & \multicolumn{2}{|c|}{$\begin{array}{c}\text { DM } \\
(n=13)\end{array}$} & \multicolumn{2}{|c|}{$\begin{array}{c}\text { DLM } \\
(n=25)\end{array}$} & \multicolumn{2}{|c|}{$\begin{array}{c}\text { CONT } \\
(n=2 I)\end{array}$} & \multirow[b]{2}{*}{ P-valor } \\
\hline & M & DP & M & DP & M & DP & $M$ & DP & \\
\hline Contar todos & 0,10 & $0,44 a b$ & 0,00 & $0,00 \mathrm{ab}$ & 0,8 & $0,08 a b$ & 0,00 & $0,00 \mathrm{ab}$ & 0,673 \\
\hline Contar maior & 2,85 & $2,92 a b$ & 3,38 & $4,19 a b$ & 5,48 & $3,50 \mathrm{a}$ & 2,00 & $2,28 b$ & 0,003 \\
\hline Contar menor & 0,25 & $0,7 \mathrm{Iab}$ & 0,00 & $0,00 \mathrm{ab}$ & 0,28 & $0,54 a b$ & 0,05 & $0,2 \mathrm{Iab}$ & 0,202 \\
\hline Contagem interna & 3,15 & $2,58 a b$ & 5,00 & $3,24 a b$ & 4,48 & $3,65 a b$ & 2,71 & $2,55 a b$ & 0,89 \\
\hline Decomposição & 2,75 & $2,42 a$ & $\mathrm{I}, 85$ & $\mathrm{I}, 72 \mathrm{ab}$ & 1,04 & $\mathrm{I}, 76 \mathrm{~b}$ & 2,19 & I,86ab & 0,037 \\
\hline Recuperação da memória & 4,75 & $3,43 a b$ & 3,77 & $3,08 \mathrm{bc}$ & 2,64 & $2,94 c$ & 7,05 & $3,30 \mathrm{a}$ & 0,000 \\
\hline
\end{tabular}

DL: alunos com dificuldades na leitura; DM: alunos com dificuldades na matemática; DLM: alunos com dificuldades na leitura e na matemática; CONT: alunos sem dificuldades de aprendizagem.

* Letras iguais indicam que as médias não diferem.

Fonte: Corso (2008).

\section{Discussão}

Os dados obtidos nas tarefas de consciência fonológica revelam que essa habilidade encontra-se em defasagem nos alunos provenientes dos grupos com DL e DL + DM. 
Tais defasagens estão relacionadas à identificação e à manipulação de fonemas, já que a consciência da sílaba não ofereceu problemas para aqueles grupos. Resultados similares têm sido apontados por outras pesquisas que demonstraram que dificuldades com a consciência fonológica, em especial a consciência fonêmica, interferem diretamente na aquisição e no domínio das correspondências letra-som necessárias a uma leitura fluente (Hecht et al., 2001).

Nas tarefas de memória fonológica para dígitos e frases, os resultados obtidos pelo grupo de alunos sem dificuldades e pelos diferentes grupos com dificuldades são bastante similares. Já na tarefa que avaliou a memória de relatos, constatou-se que os alunos com DM apresentaram um desempenho significativamente abaixo daquele evidenciado pelos alunos sem dificuldades de aprendizagem. Portanto, ficou evidenciada uma menor capacidade da memória fonológica nos alunos com DM quando a tarefa em questão exigia maior demanda cognitiva. Resultados que vão na mesma direção são apresentados por Andersson e Lyxell (2007) que evidenciaram um baixo desempenho na memória fonológica (lista de dígitos e de palavras) no grupo de alunos com DM. Os autores interpretaram esse achado como um possível atraso no desenvolvimento do componente fonológico da memória de trabalho desses alunos.

Quanto à velocidade de processamento, os alunos sem dificuldades apresentaram o menor tempo (maior velocidade) para o processamento das três tarefas propostas: letras, números e números e letras. Já os alunos com DL e DL + DM mostraram desempenho inferior naquelas tarefas, necessitando de um tempo maior (menor velocidade) para o processamento. Os alunos que apresentam DM e um bom desempenho em leitura não demonstraram desvantagem na velocidade de processamento, ao contrário, foram capazes de processar letras e números e letras com uma maior velocidade do que os alunos com DL + DM. Tais achados sugerem que o baixo desempenho em leitura está associado com a baixa velocidade de processamento, já que os alunos com $\mathrm{DL}$, independentemente do desempenho em matemática, apresentaram uma baixa velocidade de processamento. Um padrão similar de resultados foi apresentado por Willcutt et al. (2013), que encontraram dificuldades na velocidade de nomeação (tipo de medida de velocidade de processamento) no grupo de alunos com DL.

Em relação ao senso numérico, este demonstrou ser uma habilidade prejudicada no grupo de alunos com DL + DM. Embora os alunos com DM tenham apresentado desempenho inferior nessa tarefa, em relação ao grupo de controle e ao grupo com DL, tal diferença não alcançou nível de significância estatística. Possíveis justificativas para tal resultado incluem o tamanho reduzido da amostra de alunos que formou esse grupo e o ponto de corte, neste caso mais leniente, que caracterizou o instrumento utilizado nesta pesquisa (TDE) para formar a amostra de alunos com DM.

No que diz respeito à memória de trabalho, os dados indicam que o grupo com $\mathrm{DL}+\mathrm{DM}$ é capaz de armazenar uma menor quantidade de informações tanto numérica quanto não numérica na memória de trabalho, em relação aos alunos sem dificuldades. Tais resultados estão de acordo com os achados da literatura (Willcutt 
et al., 2013). Para os alunos com dificuldades apenas na leitura ou na matemática, a memória de trabalho não pareceu estar relacionada aos problemas enfrentados por tais grupos, indo de encontro a alguns resultados presentes na literatura que mostram que os alunos com DL e DM, isoladamente, demonstram problemas com a memória de trabalho (Mclean \& Hitch, 1999). Como mencionado anteriormente, é possível que esse fato tenha sido influenciado pelo tamanho da amostra, em especial do grupo com DM, que foi pequeno para revelar diferenças estatisticamente significativas em relação às tarefas de memória de trabalho propostas.

Uma possível interpretação para a não significância encontrada nessa variável, pelo grupo com DL, refere-se tanto à natureza distinta das tarefas utilizadas para avaliar a memória de trabalho quanto à divergência de instrumentos usados para selecionar os grupos com dificuldades de aprendizagem. Diferentes grupos de crianças atendem aos critérios para dificuldades na leitura e/ou na matemática, dependendo de quais instrumentos são usados para a identificação. Além disso, critérios mais lenientes ou mais restritivos acabam por formar diferentes amostras de alunos com perfis cognitivos distintos (Murphy et al., 2007). Esses aspectos acabam por gerar dados controversos e tornam a comparação e validação de resultados obtidos em diferentes pesquisas uma tarefa bastante complexa.

Quanto às estratégias utilizadas, os alunos com DM e DL + DM persistem usando aquelas menos maduras de contar nos dedos que demandam mais tempo e mais recursos da memória de trabalho, dado condizente com outras pesquisas da área (Orrantia et al., 2002). Os alunos com DL + DM utilizam com maior frequência a estratégia de contar a partir do maior, em relação aos alunos sem dificuldades.

O grupo de alunos sem dificuldades utilizou estratégias mais maduras do tipo decomposição e recuperação da memória em relação aos alunos com DM, com ou sem a presença de dificuldades na leitura. Tais resultados vão ao encontro de evidências que mostram que os alunos com DM e também aqueles com DLM utilizam o procedimento de contar nos dedos por mais tempo e não demonstram mudanças no uso de estratégias procedimentais para aquelas apoiadas na memória (Orrantia et al., 2002).

Quando os alunos foram solicitados a realizar os problemas de adição simples usando qualquer estratégia que necessitassem, não houve diferença no desempenho evidenciado pelo grupo sem dificuldades e pelos grupos com dificuldades de aprendizagem na matemática. No entanto, quando a estratégia de recuperação da memória foi posta como condição única para a resolução da tarefa, o desempenho obtido pelos alunos sem dificuldades foi superior àquele evidenciado pelos alunos com DM e DL + DM ( $p=0,003)$. Resultados que vão nessa mesma direção, apresentados por Geary et al. (2000), mostram que, quando os alunos com DM recuperam dados da memória de longo prazo, cometem muito mais erros do que aqueles sem problemas. A dificuldade para recuperar fatos da memória de longo prazo impede os alunos de desenvolver fluência no aprendizado da matemática.

Nossos resultados revelam que os diferentes grupos participantes desta pesquisa apresentam perfis cognitivos distintos. Os alunos sem dificuldades de aprendizagem 
evidenciam um melhor desempenho em relação àqueles com dificuldades, em todos os grupos de habilidades avaliadas. Para o grupo com DL, a consciência fonológica e a velocidade de processamento foram as habilidades de processamento fonológico que se mostraram mais prejudicadas. Para aqueles com DM, a memória de relatos foi a habilidade de processamento fonológico que pareceu estar em defasagem. O grupo com DL + DM foi o que se mostrou mais prejudicado, evidenciando defasagens em todas as tarefas cognitivas avaliadas.

Os diferentes perfis apresentados pelos grupos sugerem, como apontam outros estudos (Willcutt et al., 2013), que as dificuldades na leitura e na matemática são problemas distintos, mas que se correlacionam em função de fatores de risco, genéticos ou ambientais, que levam a deficiências em certas habilidades cognitivas (memória de trabalho, velocidade de processamento, memória fonológica e consciência fonológica, no caso desta pesquisa) que são fundamentais para a aprendizagem tanto da leitura quanto da matemática. No entanto, considerando que as pesquisas nessa área são recentes e apresentam resultados bastante controversos, torna-se evidente a necessidade de mais investigação.

Os resultados desta pesquisa oferecem um conjunto de implicações educacionais, entre as quais se destaca a necessidade de incluir, ao longo do ensino fundamental, tarefas escolares voltadas para o desenvolvimento do processamento fonológico e do senso numérico, habilidades ainda pouco conhecidas pelos professores da escola brasileira.

$\mathrm{O}$ estudo indicou que a consciência fonológica e a velocidade de processamento são as habilidades de processamento fonológico que se mostraram em defasagens nos alunos com DL. Com frequência, as práticas educacionais que desenvolvem as habilidades de consciência fonológica restringem-se às etapas iniciais de alfabetização, ao passo que estudos como o nosso têm apontado defasagens com tais habilidades em alunos de séries mais adiantadas, especialmente naqueles que apresentam dificuldades na leitura. Para muitas crianças, a reflexão sobre os sons da língua e as relações entre letras e sons (condições básicas a uma leitura fluente) não acontecem como consequência natural de suas interações com variadas práticas de leitura. Para essas crianças, é necessária a valorização do ensino explícito da competência linguística.

A pesquisa sugere também que o ensino da matemática dê ênfase maior ao desenvolvimento do senso numérico. De fato, constatamos dificuldades com o senso numérico nos alunos com DL + DM, mas não naqueles com apenas DM. É importante destacar, entretanto, que esse resultado não exclui a possibilidade de que, para muitos alunos, um senso numérico pouco desenvolvido esteja na base de suas dificuldades em matemática. A recuperação de fatos aritméticos da memória, por sua vez, exerce um importante papel no desenvolvimento de processos matemáticos mais complexos (por exemplo, cálculo de multidígitos e solução de problemas), e, por isso, a importância de se dar mais ênfase ao ensino dessa habilidade principalmente para os alunos com dificuldades na matemática. Como vimos neste trabalho, a recuperação de fatos da memória parece estar associada a habilidades cognitivas do tipo memória de trabalho e velocidade de processamento, o que sugere que tais processos também sejam estimulados. 
Outra implicação que o estudo oferece para o ensino diz respeito à importância de distinguirmos os diferentes grupos de alunos com dificuldades de aprendizagem. Vimos que os alunos com DL + DM apresentam defasagens que abrangem as diversas habilidades avaliadas. Para esse grupo de alunos, a intervenção requer uma soma de esforços: intervenção sistemática e intensiva que englobe adaptação curricular e planejamento individualizado. Talvez, para os alunos que evidenciam dificuldades somente na leitura ou na matemática, a intervenção não necessite ser tão intensa, mas necessariamente requer uma constante reflexão do professor sobre sua intervenção pedagógica, obrigando-o a uma flexibilização constante para adaptar seu ensino ao estilo de aprender do aluno, atendendo, portanto, às suas características e necessidades individuais.

As pesquisas que se dedicam ao estudo das habilidades cognitivas subjacentes às aprendizagens da leitura e da matemática são relativamente recentes, e, como consequência, suas aplicações para as práticas educacionais são ainda escassas. Tais pesquisas, no entanto, são fundamentais para que possamos avançar nos processos de prevenção de dificuldades. Ao identificarmos que sistemas cognitivos encontram-se deficitários nos alunos com dificuldades de aprendizagem, estaremos mais capacitados para criar intervenções que possam evitar que os alunos em risco venham a desenvolver problemas futuros.

\section{Referências}

Andersson, U., \& Lyxell, B. (2007).Working memory deficit in children with mathematical difficulties: a general or specific deficit? Journal of Experimental Child Psychology, 96(3), 197-228.

Ashkenazi, S., Black, J. M., Black, D. A., Hoeft, F., \& Menon, V. (2013). Neurobiological underpinnings of math and reading learning disabilities. Journal of Learning Disabilities, 46(6), 549-569.

Baddeley, A. D., \& Hitch, G. J. (1974). Working memory. In G. H. Bower (Ed.). The psychology of learning and motivation (pp. 47-91). London: Academic Press.

Cardoso-Martins, C., \& Pennington, B. (2001). Qual é a contribuição da nomeação seriada rápida para a habilidade de leitura e escrita? Evidência de crianças e adolescentes com e sem dificuldades de leitura. Psicologia Reflexão e Crítica, 14(2), 387-397.

Corso, L. V. (2008). Dificuldades na Leitura e na Matemática: um estudo dos processos cognitivos em alunos da $3^{a}$ a $6^{a}$ série do Ensino Fundamental. 218f. Tese de doutorado, Faculdade de Educação, Universidade Federal do Rio Grande do Sul, Porto Alegre, Rio Grande do Sul, RS, Brasil. 
Dyson, N., Jordan, N., \& Glutting, J. (2011). A number sense intervention for lowincome kindergartners at risk for mathematics difficulties. Journal of Learning Disabilities, Chicago, 42(2), 166-181.

Geary, D. C., Hamson, C. O., \& Hoard, M. K. (2000). Numerical and arithmetical cognition: a longitudinal study of process and concept deficits in children with learning disabilities. Journal of Experimental Child Psychology, 77, 236-263.

Gersten, R., Jordan, N., \& Flojo, J. (2005). Early Identification and Interventions for students with mathematics difficulties. Journal of Learning Disabilities, 38(4), 293-304.

Golbert, C. S. (1988). A evolução psicolingüística e suas implicações na alfabetização: teoria, avaliação, reflexões. Porto Alegre: Artmed.

Hecht, S., Torgesen, J., Wagner, R., \& Rashotte, C. (2001). The relations between phonological processing abilities and emerging individual differences in mathematical computation skills: a longitudinal study from second to fifth grades. Journal of Experimental Child Psychology, 79, 192-227.

Hopkins, S. L., \& Lawson, M. J. (2006). The effect counting speed has on developing a reliance on retrieval in basic addition. Contemporary Educational Psychology, $31,208-227$.

Mclean, J. F., \& Hitch, G. J. (1999). Working memory impairments in children with specific arithmetic learning difficulties. Journal of Experimental Child Psychology, 74, 240-260.

Moojen, S., Lamprecht, R., Santos, R., Freitas, G., Brodacz, R., Siqueira M., Costa, A., \& Guarda, E. (2003). Confias - consciência fonológica: instrumento de avaliação seqüencial. São Paulo: Casa do Psicólogo.

Murphy, M. M., Mazzocco, M. M. M., Hanich, L. B., \& Early, M. C. (2007). Cognitive characteristics of children with mathematics learning disability (MLD) vary as a function of the cutoff criterion used to define MLD. Journal of Learning Disabilities, 40(5), 458-478.

Okamoto, Y., \& Case, R. (1996). Exploring the microstructure of children's central conceptual structures in the domain of number. Monographs of the Society for Research in Child Development, Chicago, 61, 27-59.

Orrantia, J., Martínez, J., Morán, M., \& Fernández, J. (2002). Dificultades en el aprendizaje de la aritmética: un analisis desde los modelos cronométricos. Cognitiva, 14(2), 183-201.

Shaywitz, S. (2006). Entendendo a dislexia. Porto Alegre: Artmed.

Stein, L. (1994). TDE - teste de desempenho escolar: manual para a aplicação e interpretação. São Paulo: Casa do Psicólogo. 
Swanson, H. L. (1999). Reading comprehension and working memory in learning disabled readers: is the phonological loop more important than the executive system? Journal of Experimental Child Psychology, 72, 1-31.

Willcutt, E. G., Petrill, S. A., Wu, S., Boada, R., DeFries, J. C., Olson, R. K., \& Pennington, B. F. (2013). Comorbidity between reading disability and math disability: concurrent psychopathology, functional impairment, and neuropsychological functioning. Journal of Learning Disabilities, 46(6), 500-516.

Yuill, N., Oakhill, J., \& Parkin, A. (1989). Working memory, comprehension ability and the resolution of text anomaly. British Journal of Psychology, 80, 351-361.

Submissão: 29.4.2014

Aceitação: 31.3 .2015 